\section{Elevated irisin and alanine aminotransferase levels in hypothyroidism: role of oxidative stress?}

Bruno Halpern'

$I^{\prime}$ n this issue of Archives of Endocrinology and Metabolism, two articles help to increase our knowledge about the connections between thyroid status and energy metabolism. Although the relation between thyroid disease with obesity and metabolic dysfunction has been well established for many decades, some of their linking mechanisms are surprisingly still poorly understood.

In the first paper, a cross-sectional observational study, Ates and cols. (1) measured irisin levels of 37 patients with newly diagnosed hypothyroidism (and a mean TSH of $13.1 \mathrm{mIU} / \mathrm{L}$ ) and 37 healthy volunteers.

Irisin is a recently identified proliferator-activated receptor-gamma coactivator- $1 \alpha$ (PGC-1 $\alpha$ )-dependent myokine that drives browning of white fat via elevation of uncoupling protein-1 (UCP-1), leading to thermogenesis and metabolic improvement (2). It was demonstrated to be a cleavage product of the extracellular portion of type I membrane protein fibronectin type III domain containing 5 (FNDC5), that is released after physical activity. The isolation of irisin in 2012 led to a great rush in understanding its function and metabolic relevance, as it was initially considered one of the main drivers of the beneficial effects of exercise $(2,3)$. However, its impact was weakened by further studies that questioned the metabolic relevance of irisin in humans since there is a substantial controversy whether humans are able to cleave the protein FNDC5 $(4,5)$. This would happen due to humans have a truncated form of FNDC5 gene, and as a result, irisin levels be insignificant, and its detection in serum would be due to analytical interferences. Even though this theory is up to this time a matter of debate, many publications about irisin have arisen in the last years, both in animals and humans.

Another disappointment about the potential role of irisin in metabolic improvement (and maybe as a potential therapeutic agent) was the observation that obese individuals and patients with metabolic syndrome have higher levels of irisin and that weight loss (regardless of whether through caloric restriction or bariatric surgery) leads to a decrease in irisin levels (6-8). Could this phenomenon be due to an "irisin resistance" analogous to insulin or leptin resistance or it could be due to a defense against metabolic impairment? These are questions still unanswered, and the first study in this journal issue points in the same controversial direction, demonstrating higher levels of irisin in hypothyroid patients (1). In the study, the mean BMI was not statistically different between groups $\left(27.77 \mathrm{vs} 26.11 \mathrm{~kg} / \mathrm{m}^{2}\right.$, in hypothyroid and control groups, respectively) and the main parameters associated with irisin levels were age, TSH and free T4 levels. On one hand, these results are in agreement with a study in a murine model of thyroid dysfunction, in which an upregulation of irisin levels in both hyper and hypothyroid rats, as well as a positive correlation between irisin and
1 Grupo de Obesidade e Síndrome Metabólica, Disciplina de Endocrinologia e Metabologia, Hospital das Clínicas da Faculdade de Medicina da Universidade de São Paulo (HC-FMUSP), São Paulo, SP, Brasil

Correspondence to: Bruno Halpern brunohalpern@hotmail.com

Received on Apr/8/2016 Accepted on Apr/8/2016 DOI: 10.1590/2359-3997000000166 
creatine kinase (CK) levels were observed. The authors concluded that the muscle oxidative stress present in both conditions could possibly be responsible for these results (9), which were corroborated by increased irisin levels in statin-treated patients (10). Acute exercise elevated irisin levels in all groups of thyroid dysfunction rats, although chronic training exercise did not, which provides consistency to the notion that irisin might possibly be secreted as a result of muscle acute stress, decreasing the likelihood of being a major factor of the eumetabolic status of physically active patients (11). On the other hand, a cross-sectional human study with newly diagnosed hyper or hypothyroid patients found a negative relation between irisin and TSH levels, as well as between irisin and CK levels, with higher irisin levels in the hyperthyroid group. However, in contrast to the first article in this issue, there was not a control group of subjects with normal thyroid function (12). Since hyperthyroidism is also associated with muscle stress, these results can be in agreement with a rise in irisin levels due to muscle damage. Clearly, the field has yet many gaps, but these data gives us insight of irisin being more an oxidative stress marker than a metabolic protective hormone.

In the second manuscript in this AE\&M issue, also a transversal analysis data study, Silva and cols. evaluate the relationship of slightly increased alanine aminotransferase (ALT) levels (above the upper tertile) with thyroid diseases (13), mainly chronic lymphocytic thyroiditis. Although nearly $90 \%$ of the included patients took oral doses of levothyroxine, $52 \%$ presented TSH above $3.0 \mathrm{mIU} / \mathrm{L}$, and of these subjects, more than $65 \%$ had ALT levels in the upper tertile (compared to $44.6 \%$ in individuals with normal TSH, statistically significant in bivariate and multivariate analysis). A positive correlation was also found between higher ALT and both systemic arterial hypertension and abdominal circumference. Although a relationship of overt hyperthyroidism with ALT levels and liver function is well documented, studies showing association with hypothyroidism are infrequent and, in this case, the link between higher ALT and TSH levels most probably reflects non-alcoholic fatty liver disease or non-alcoholic steatohepatitis (NAFLD/NASH), as suggested by the presence of metabolic syndrome components (systemic arterial hypertension and increased abdominal circumference). Even though ALT level is often considered an easily accessible surrogate marker for evaluating underlying liver disease activity and severity of liver injury controversies remains about the prognostic value of ALT levels in determine NAFLD progression to NASH (since patients with normal ALT levels can reveal in the liver biopsy an advanced liver disease) $(14,15)$. Nevertheless, there is indeed evidence of ALT as a prognostic factor to the development of type 2 diabetes and cardiovascular disease (16). Silva and cols. results are in concordance with several other studies that evaluated the relationship of both hypothyroidism and NAFLD/ NASH. A linear relationship between TSH levels and NAFLD (diagnosed both by abnormal liver tests and ultrasonography) was reported by Chung and cols. even in upper normal TSH levels and independently of any other known risk factor (17). In this study, there were a $40 \%$ increased risk of NAFLD in individuals with TSH between 2.5-4.5 mIU/L compared to individuals with TSH lower than $2.5 \mathrm{mIU} / \mathrm{L}$, which still yield more controversy regarding the never-ending discussion of whether to treat or not subclinical hypothyroidism. A systematic review found a $15.2 \%$ to $36.3 \%$ prevalence of hypothyroidism in patients with NAFLD/NASH and concluded that hypothyroidism should be added to risk factors of NAFLD/NASH (18). What remains to be known is if hypothyroidism predicts or not the severity of fatty liver disease, since different studies yielded controversial results (17-20). Interestingly, however, is the possible role of oxidative stress and mitochondrial dysfunction in the pathogenesis of NASH. In parallel with what occurs in muscle, hypothyroidism could also have a role in hepatic oxidative stress. For example, several markers of oxidative stress as serum malonildialdehyde, a lipid peroxidation marker, as well as reactive oxygen species and nitric oxide have been shown to be elevated in hypothyroidism $(21,22)$.

In conclusion, the possible role of oxidative stress could be a point of contact between both studies in this journal issue. Hypothyroidism, wrongly considered to be a major cause of weight gain due to decreased energy expenditure by the general public, apparently has a more subtle effect on metabolic impairment at the intracellular level, and although there may be a lot of causes, since thyroid hormones are ubiquitous in the body, oxidative damage should not be discarded as a possible explanation for higher irisin and ALT levels observed in these studies.

Disclosure: no potential conflict of interest relevant to this article was reported. 


\section{REFERENCES}

1. Ateş I, Altay M, Topçuoğlu C, Yilmaz FM. Circulating levels of irisin is elevated in hypothyroidism, a case-control study. Arch Endocrinol Metab. 2016;60(2):95-100.

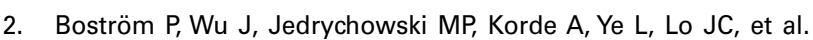
A PGC1- $\alpha$-dependent myokine that drives brown-fat-like development of white fat and thermogenesis. Nature. 2012;481(7382): 463-8.

3. Pedersen BK. A muscular twist on the fate of fat. $\mathrm{N}$ Engl $\mathrm{J}$ Med. 2012;366(16):1544-5.

4. Raschke S, Elsen M, Gassenhuber H, Sommerfield M, Schwahn $U$, Brockmann B, et al. Evidence against a beneficial effect of irisin in humans. PLoS One. 2013;8(9):e73680.

5. Crujeiras AB, Pardo M, Casanueva FF. Irisin: 'fat' or artefact. Clin Endocrinol (Oxf). 2015;82(4):467-74.

6. Park KH, Zaichenko L, Brinkoetter M, Thakkar B, Sahin-Efe A, Joung $\mathrm{KE}$, et al. Circulating irisin in relation to insulin resistance and the metabolic syndrome. J Clin Endocrinol Metab. 2013;98(12):4899-907.

7. Stengel A, HofmannT, Goebel-Stengel M, Elbelt U, Kobelt P, Klapp BF. Circulating levels of irisin in patients with anorexia nervosa and different stages of obesity--correlation with body mass index. Peptides. 2013;39:125-30.

8. Huh JY, Panagiotou G, Mougios V, Brinkoetter M, Vamvini MT, Schneider BE, et al. FNDC5 and irisin in humans: I. Predictors of circulating concentrations in serum and plasma and II. mRNA expression and circulating concentrations in response to weight loss and exercise. Metabolism. 2012;61(12):1725-38.

9. Samy DM, Ismail CA, Nassra RA. Circulating irisin concentrations in rat models of thyroid dysfunction -- effect of exercise. Metabolism. 2015;64(7):804-13.

10. Gouni-Berthold I, Berthold GK, Huh JY, Berman R, Spenrath N, Krone W, et al. Effects of lipid-lowering drugs on irisin in human subjects in vivo and in human skeletal muscle cells ex vivo. PLoS One. 2013;8(9):e72858.

11. Huh JY, Montzoros C. Irisin physiology, oxidative stress, and thyroid dysfunction: What next? Metabolism. 2015;64(7):765-7.
12. Ruchala M, Zybek A, Szczepanek-Parulska E. Serum irisin levels and thyroid function--newly discovered association. Peptides. 2014;60:51-5.

13. Silva NO, Ronsoni MF, Colombo BS, Correa CG, Hatanaka SA, Canalli MHBS, et al. Clinical and laboratory characteristics of patients with thyroid diseases with and without alanine aminotransferase levels above the upper tertile - Cross-sectional analytical study. Arch Endocrinol Metab. 2016;60(2):101-7.

14. Verma S, Jensen D, Hart J, Mohanty SR. Predictive value of ALT levels for non-alcoholic steatohepatitis (NASH) and advanced fibrosis in non-alcoholic fatty liver disease (NAFLD). Liver Int. 2013;33(9):1398-405.

15. Amarapurka DN, Amarapurkar AD, Patel ND, Agal S, Baigal R, Gupte P, et al. Nonalcoholic steatohepatitis (NASH) with diabetes: predictors of liver fibrosis. Ann Hepatol. 2006;5(1):30-3.

16. Goessling W, Massaro JM, Vasan RS, D'Agostino RB, Ellison RC, Fox CS. Aminotransferase levels and 20-year risk of metabolic syndrome, diabetes, and cardiovascular disease. Gastroenterology. 2008;135(6):1935-44

17. Chung GE, Kim D, Kim W, Yim JY, Park MJ, Kim YJ, et al. Nonalcoholic fatty liver disease across the spectrum of hypothyroidism. J Hepatol. 2012;57(1):150-6.

18. Eshraghian A, Jahromi AH. Non-alcoholic fatty liver disease and thyroid dysfunction: A systematic review. World J Gastroenterol. 2014;20(25):8102-9.

19. Pagadala MR, Zein CO, Dasarathy S, Yerian LM, Lopez R, McCullough AJ. Prevalence of hypothyroidism in nonalcoholic fatty liver disease. Dig Dis Sci. 2012;57(2):528-34.

20. Mazo DF, Lima VM, Stefano JT, Rabelo F, Faintuch J, Oliveira CP. Gluco-lipidic indices in treated hypothyroidism associated with nonalcoholic fatty liver disease. Arq Gastroenterol. 2011;48(3):186-9.

21. Torun AN, Kulaksizoglu S, Kulaksizoglu M, Pamuk BO, Isbilen E, Tutuncu NB. Serum total antioxidant status and lipid peroxidation marker malondialdehyde levels in overt and subclinical hypothyroidism. Clin Endocrinol (Oxf). 2009;70:469-74.

22. Baskol G, Atmaca H, Tanriverdi F, Baskol M, Kocer D, Bayram F. Oxidative stress and enzymatic antioxidant status in patients with hypothyroidism before and after treatment. Exp Clin Endocrinol Diabetes. 2007;115(8):522-6. 